\title{
EFFECT OF WALE-WISE INCREASED TUCK AND MISS LOOPS ON SPIRALITY OF SINGLE JERSEY KNIT FABRICS
}

\author{
Md. Azharul Islam ${ }^{1}$ \\ ${ }^{\text {l} L e c t u r e r, ~ D e p a r t m e n t ~ o f ~ T e x t i l e ~ E n g i n e e r i n g, ~ D a f f o d i l ~ I n t e r n a t i o n a l ~ U n i v e r s i t y, ~ D h a k a, ~ B a n g l a d e s h ~}$
}

\begin{abstract}
Derivatives of single jersey can be produced by combining the tuck and miss loops in both wale and course direction. But wale wise increment of tuck and miss loops are mostly practiced. This paper focused on spirality which is a common fault of knit fabrics. The wale wise increment of tuck and miss loops, individually have effects on spirality. Total nine single jersey derivatives were knitted to observe the changes. From total study it was perceived with the increment of tuck and miss loops spirality increases and higher spirality was found for miss loop fabrics $(10 \%, 12.8 \%, 4.8 \%$ and $6.3 \%)$ than tuck loop fabrics $(2 \%, 4 \%, 0.9 \%$ and $2 \%)$. It was also found that an all knit course insertion on pique and cross miss designs reduce the spirality almost to half for lacoste and locknit designs. It means that higher presence of knit loops can reduce the spirality.
\end{abstract}

Keywords: Tuck loop, Miss Loop, spirality, and single jersey derivatives

\section{INTRODUCTION}

Spirality is a default problem of knit fabrics which is produced in circular knitting machines. It is obvious for wale and course to be perpendicular with each other. The displacement of this wale and course causes spirality in knit fabrics. The total work done on single jersey derivatives by increasing the tuck and miss loops in wale direction to observe the changing pattern of spirality.

Islam A. et al. [1] studied the effect of machine gauge on the spirality of single jersey knit fabrics. They finally showed that lower spirality was found for 24 machine gauge. This paper was focused on the products from Bangladesh perspectives. Araujo and Smith [2,3] studied the effect of machine, yarn and fabric properties on the fabric spirality. Their observation on spirality finally declares that spirality depends on machine cut, feed density, machine rotation direction, loop shape, yarn twist value (twist liveliness) and yarn twist direction. They suggested to use S-twist yarns in machines rotating counterclockwise and Z-twist yarn in machines rotating clockwise. Plied yarns, plating techniques and yarns with different twist directions can be used to solve or reduce this problem. They also presented an empirical model to predict fabric spirality on the fabric.

In a recent journal paper, Singh $G$ et al. [4] confirmed the variable factors, namely linear density, twist factor, machine gauge and stitch length, influence the shrinkage to a variable degree, stitch length is the dominating factor. If shrinkage can be controlled, fabric weight is predicted with high accuracy.

M.A. Shahid et al. [5] produced Single jerseys knitted fabrics were produced from different yarn count $(26 \mathrm{~s} / 1,28 \mathrm{~s} / 1$, and $30 \mathrm{~s} / 1)$ using different stitch length $(2.58 \mathrm{~mm}, 2.63 \mathrm{~mm}, 2.68 \mathrm{~mm}$,
$2.70 \mathrm{~mm}$ and $2.73 \mathrm{~mm}$ ) with positive feed device in Jiunn long knitting machine. The fabrics were dyed in light shade by a winch dyeing machine, dried with Unitech Stenter machine and compacted by Ferraro compactor using selected parameters. The results showed that spirality\% were lowest for 26/1 Ne, 28/1 Ne and 30/1 Ne knitted fabrics at stitch length $4.58 \mathrm{~mm}, 2.70 \mathrm{~mm} \& 2.73 \mathrm{~mm}$ before compacting and at stitch length $2.68 \mathrm{~mm}, 2.68 \mathrm{~mm} \quad \& \quad 2.73 \mathrm{~mm}$ after compacting respectively.

More experimental studies [6-10] have explored the different contributory factors on spirality. Some are machine related like use of multiple feeders and gauge, whereas some are associated with constituent yarns like twist liveliness and linear density. Essentially in almost all citations, it has been clearly demonstrated that it is the relaxation of torsional stresses which causes the dimensional distortions and instability in the knitted loop construction. This leads to the appearance of spirality in the fabrics. The distortion of loops progresses till a fully relaxed state is reached by subjecting the fabric to repeated washing, rinsing and tumble drying. This state has been referred to as 'reference state' by Heap et al. (11]

\section{MATERIAL AND METHOD}

Tuck and miss loops are used to diversify the single jersey knit fabrics. To conduct this work tuck and miss loops were individually increased in wale direction to create single jersey derivatives and it was dyed on dyeing machine and finished with tube dryer and tube compactor. Total nine single jersey derivatives were knitted with $30 \mathrm{Ne}$ yarn on a 21" diameter-24 gauge knitting machine (Pailung, Taiwan) and processed to study the spirality. The notation diagrams of all the knitted samples are given below: 


\subsection{Single Jersey Plain}

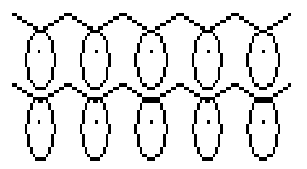

Fig 1: Notation diagram of Single Jersey (plain)

\subsection{Single Cross Tuck / Single Pique}

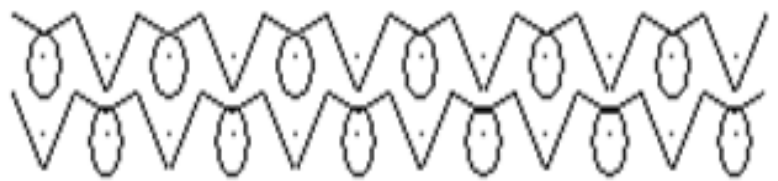

Fig 2: Notation diagram of single pique

\subsection{Double Cross Tuck / Double Pique}

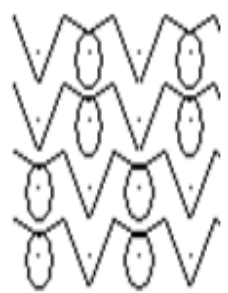

Fig 3: Notation diagram of Double Pique

\subsection{Single Cross Miss}

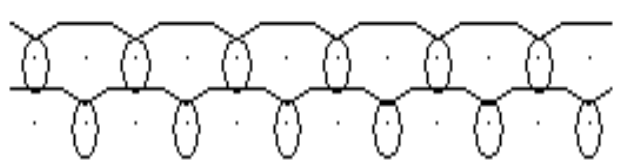

Fig 4: Notation diagram of Single Cross Miss

\subsection{Double Cross Miss}

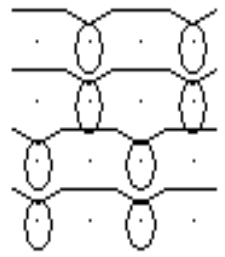

Fig 5: Notation diagram of Double Cross Miss

\subsection{Single Lacoste}

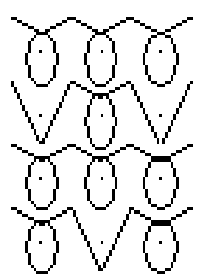

Fig 6: Notation diagram of Single Lacoste

\subsection{Double Lacoste}

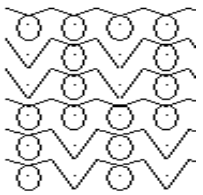

Fig 7: Notation diagram of Double Lacoste

\subsection{Single Locknit}

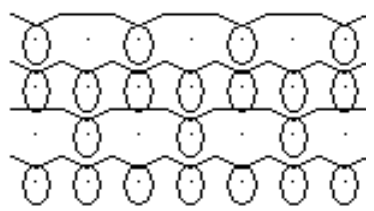

Fig 8: Notation diagram of single locknit

\subsection{Double Locknit}

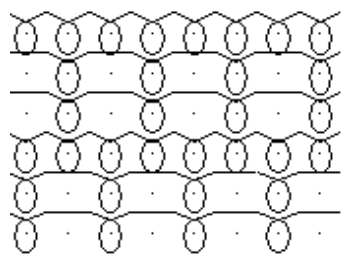

Fig 9: Notation diagram of double locknit

Each samples were knitted for $5 \mathrm{~kg}$ and total $45 \mathrm{~kg}$ fabrics were dyed on Fong's dyeing machine (origin: Taiwan). A suitable dye recipe for light colour (light pink) was selected to dye all the samples. After dyeing samples were dried at Dilmenler tube dryer (origin: Turkey) having $10 \%$ overfeed, $130^{\circ} \mathrm{C}$ temperature, machine speed $=8$ and compacted at Tubetex tube compactor (origin: USA) with $95 \%$ overfeed, $8 \%$ compaction, $25 \mathrm{rpm}$ and width was set at $20 \mathrm{inch}$.

After finishing, sample was prepared for spirality test. Spirality or twisting in a garment is appeared after washing. As a result one of the side seams comes at front of the garment when wearer wears it. Spirality percentage depends on fabric torque and garment structure.

\subsection{Procedure of Spirality Test:}

By the following way we can test spirality:

\subsubsection{Sample:}

Two piece of $50 \mathrm{~cm} \times 50 \mathrm{~cm}$ fabric is taken for test.

i. Conditioning: put the sample in the table for 4 hours conditioning before starting test.

ii. Cut the sample $50 \mathrm{~cm} \times 50 \mathrm{~cm}$ and benchmark should be $35 \mathrm{~cm} \times 35 \mathrm{~cm}$. Stitch the sample (3 sides).by over lock sewing machine.

iii. Sample is washed in washing machine.

iv. All samples are dried on line dryer. 


\subsubsection{Spirality Calculation:}

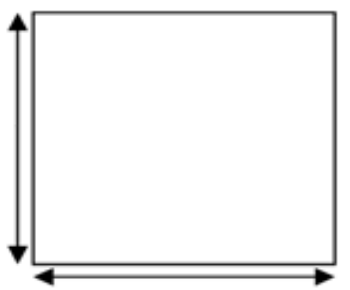

S

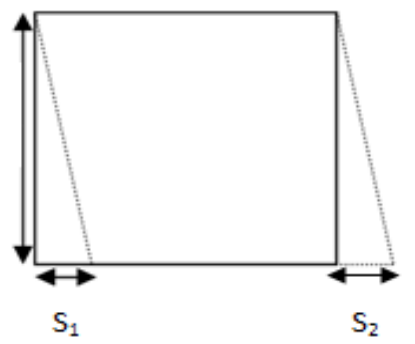

Fig 10: Spirality

Total length $\mathrm{S}=47 \mathrm{~cm}$

Twist in one end $\mathrm{S} 1=2.3 \mathrm{~cm}$

Twist in another end $\mathrm{S} 2=2.2 \mathrm{~cm}$

Average Spirality $=(2.3+2.2) / 2=2.25 \mathrm{~cm}$

So, Spirality $=\frac{2.25}{47} * 100=4.78 \%$

By following the above procedure spirality was tested for all the nine samples and the obtained values are enlisted below:

\subsection{Effect of tuck loops on spirality:}

Table 1: Effect of tuck loops on spirality of pique and lacoste

\begin{tabular}{|l|l|l|}
\hline \multirow{2}{*}{ Fabric name } & $\begin{array}{l}\text { Count } \\
(\mathrm{Ne})\end{array}$ & Spirality \\
\cline { 3 - 3 } & After Wash\% \\
\hline Single Jersey & 30 & 1.00 \\
\hline Single Pique & 30 & 2 \\
\hline Double Pique & 30 & 4 \\
\hline Single Lacoste & 30 & 0.9 \\
\hline Double Lacoste & 30 & 2 \\
\hline
\end{tabular}

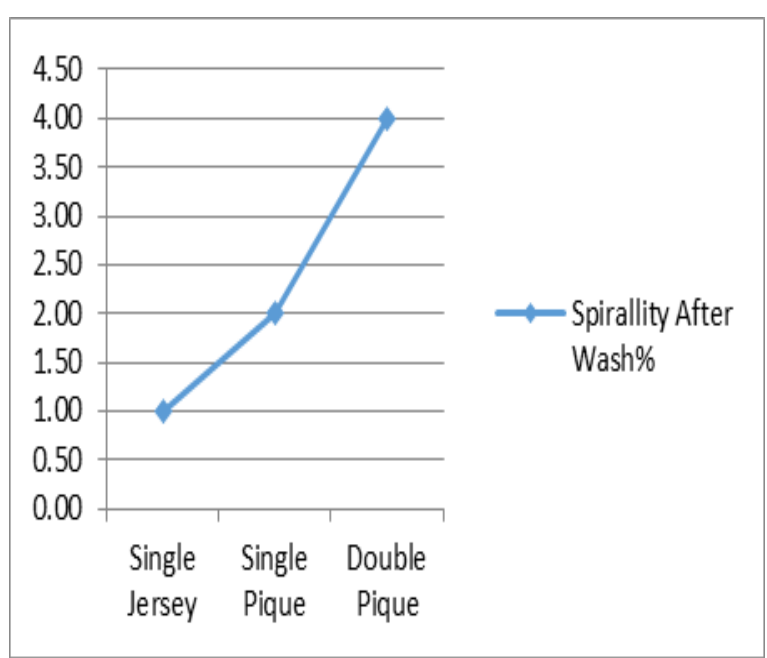

Chart 1: Effect of tuck loops on spirality of pique

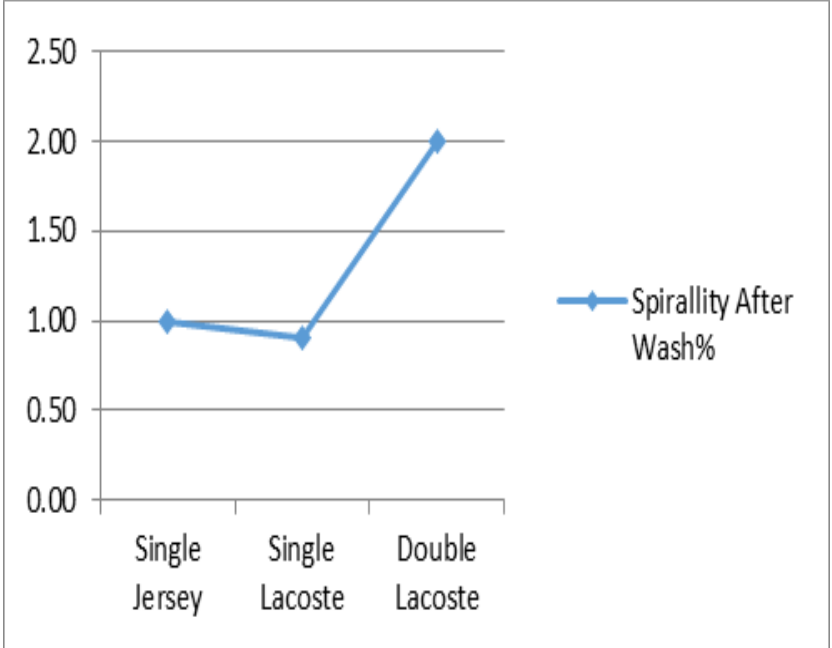

Chart 2: Effect of tuck loops on spirality of lacoste

\subsection{Effect of miss loops on spirality:}

Table 2: Effect of miss loops on spirality of cross miss and locknit

\begin{tabular}{|l|l|l|}
\hline \multirow{2}{*}{ Fabric name } & $\begin{array}{l}\text { Count } \\
(\mathrm{Ne})\end{array}$ & Spirality \\
\cline { 3 - 3 } & 30 & After Wash\% \\
\hline Single Jersey & 300 \\
\hline Single cross miss & 30 & 10 \\
\hline Double cross miss & 30 & 12.8 \\
\hline Single locknit & 30 & 4.8 \\
\hline Double locknit & 30 & 6.3 \\
\hline
\end{tabular}

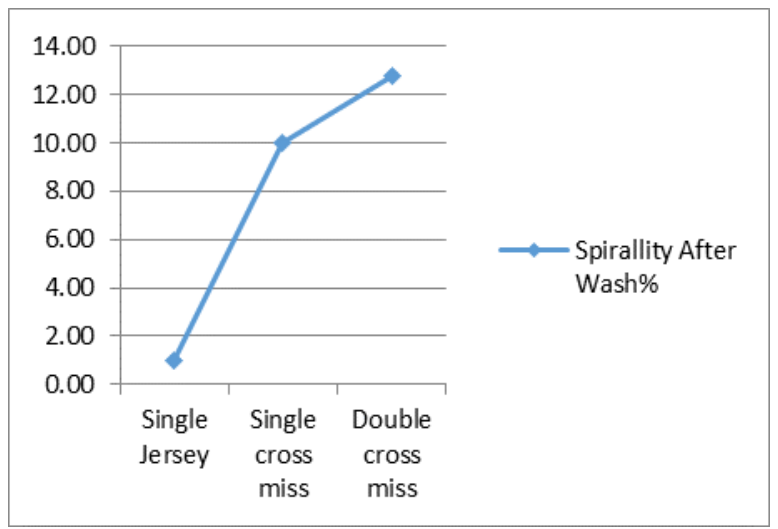

Chart 3: Effect of miss loops on spirality of cross miss 


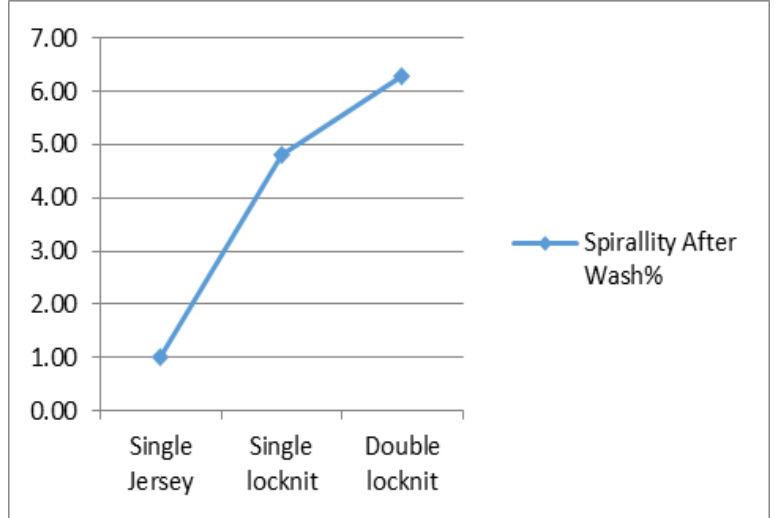

Chart 4: Effect of miss loops on spirality of locknit

\section{RESULTS AND DISCUSSION}

Table 1 , chart $1 \&$ chart 2 show that single pique (2\%) has double spirality than single lacoste $(0.9 \%)$ and double pique (4\%) has twice spirality than double lacoste $(2 \%)$. It means that all knit course insertion on pique design reduces the spirality to half compared with cross tuck designs.

From table 2, chart $3 \&$ chart 4 , it is observed that the spirality\% is very high for cross miss structure than locknit structure. For both structures spirality increase with the increasing of miss loop involvement For cross miss designs spirality was $10 \%$ and $12.8 \%$ (single cross miss and double cross miss) whereas locknit designs spirality was $4.8 \%$ and $6.3 \%$ (single locknit and double locknit). Finally it can be discussed that insertion of all knit courses decreases the spirality of cross miss design fabrics which almost half for locknit designs than cross miss.

By comparing Table 1 and table 2, it can be conferred that pique \& lacoste fabrics have lower spirality than cross miss \& locknit designs. It means that presence of miss loop increases the spirality than tuck loop.

\section{CONCLUSIONS}

This paper finally reveals that the tuck and miss loops have very definable effect on spirality of knit fabrics. By fixing the entire machine and processing parameters the total works have been carried out and it can be concluded as the below:

1. Pique fabrics have double spirality than lacoste fabrics as well as with the wale wise increment of tuck loops spirality increases.

2. Cross miss fabrics have twice spirality than locknit fabrics and spirality increases with the increment of miss loops in wale direction

3. Tuck loop fabrics have lower spirality than that of tuck loop fabrics.

\section{ACKNOWLEDGEMENTS}

The author gratefully acknowledges the help \& support from Impress-Newtex Composite Textile Ltd, Gorai, Mirzapur, Tangail, Bangladesh.

\section{REFERENCES}

[1] Md. Azharul Islam, Abu Naser Md. Ahsanul Haque, Selection of suitable machine gauge by considering the GSM, shrinkage and spirality of single jersey knit fabrics, The International Journal's Research Journal of Science \& IT management, Volume: 3, Number: 3 Jan2014, pp: 50-55

[2] M.D. de Araujo, G.W. Smith, Spirality of Knitted Fabrics, Part I: The Nature of Spirality, Textile Research Journal, 1989, vol. 59, 247-256.

[3] M.D. de Araujo, G.W. Smith, Spirality of Knitted Fabrics, Part II: The Effect of Yarn Spinning Technology, Textile Re-search Journal, 1989, vol. 59, 350-356.

[4] G. Singh, K Roy, R Varshney \& A Goyal, Dimensional parameters of single jersey cotton knitted fabrics, Indian Journal of Fibre \& Textile Research, Vol-36, june 2011, pp: 111-116

[5] M.A.Shahid, F.Ahmed, A.K.M.Mahabubuzzaman, M.A.Hannan \& A.N.Khan , Spirality in cotton knit fabrics before and after compacting using selected yarn count and stitch length, J. Innov. Dev.Strategy4(2):1117(December2010).

[6] De araujo M D \& Smith G W, Spirality of Knitted Fabrics, Part I: The Nature of Spirality, Text Res. J, 59 (1989) 247.

[7] Davis W \& Dewards C H, J Text Inst, 25 (1934) T122

[8] Lord P R, Mohamed M H \& Ajgaonkar D B, Text Res. J, 44 (1974) 405.

[9] Oinuma R \& Takeda H, J Text Mech Soc Japan (Eng edn), 34 (3) (1988) 74

[10] Banerjee P K \& Alaiban T S, Text Res. J, 58(1988) 287.

[11] Heap S A, Greenwood P F, Leah R D, Eaton J T, Stevens J C \& Keher P, Text res. J 53(1983) 109

\section{BIOGRAPHIE}

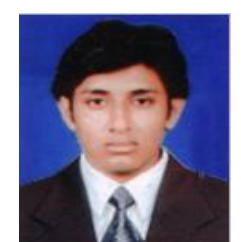

Md. Azharul Islam completed his graduation from College of Textile Technology, University of Dhaka. His interest area in textile is fabric manufacturing technology. He has teaching experience over 4 years and currently working at Daffodil International University. 